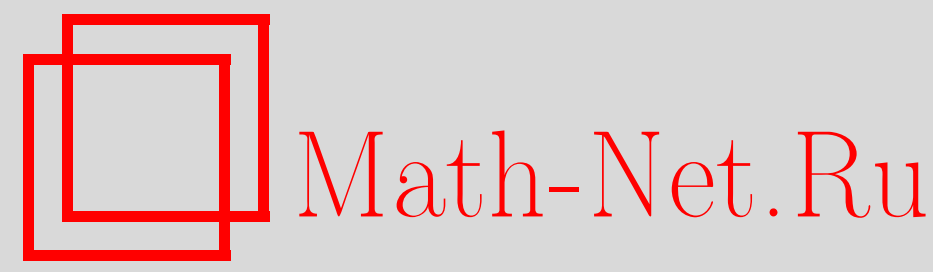

К. А. Зубрилин, О наибольшем нильпотентном идеале в алгебрах, удовлетворяющих тождествам Капелли, Матем. сб., 1997, том 188, номер 8, 93-102

DOI: https://doi.org/10.4213/sm253

Использование Общероссийского математического портала Math-Net.Ru подразумевает, что вы прочитали и согласны с пользовательским соглашением

http://www . mathnet.ru/rus/agreement

Параметры загрузки:

IP: 54.198 .187 .58

26 апреля 2023 г., 03:06:10 
УДК 512

\author{
К.А. Зубрилин
}

\title{
О наибольшем нильпотентном идеале в алгебрах, удовлетворяющих тождествам Капелли
}

\begin{abstract}
Доказано, что во всякой конечно порожденной алгебре конечной сигнатуры над произвольным полем или нётеровым коммутативно-ассоциативным кольцом, удовлетворяющей тождествам Капелли некоторого порядка, существует наиболњший нильпотентный идеал.

Библиография: 7 названий.
\end{abstract}

Говорят, что алгебра удовлетворяет системе тожсдеств Kапелли порядка n, если всякий полином, полилинейный и кососимметричный по некоторым $n$ своим переменньм, является тождеством этой алгебры.

Тождества Капелли могут быть определены для произвольной сигнатуры, хотя первоначально тождества Капелли рассматривались только для ассоциативных алгебр [1].

Тождества Капелли, с одной стороны, являются естественным обобщением тождества коммутативности и тождества ассоциативности. Если бинарная алгебра удовлетворяет тождествам Капелли порядка 2, то эта алгебра коммутативна и ассоциативна. С другой стороны, выполнение тождеств Капелли может рассматриваться как обобшение свойства конечномерности алгебры, поскольку во всякой $m$-мерной алгебре выполняются тождества Капелли порядка $m+1$.

Впервые тождества Капелли были введены в рассмотрение Ю. П. Размысловым в [1] в связи с решением проблемы радикала ассоциативной РІ-алгебры.

Комбинаторные аспекты тождеств Капелли произвольной сигнатуры изучались Размысловьм в [2]. В этой же работе была построена структурная теория алгебр произвольной конечной сигнатуры над полем нулевой характеристики, удовлетворяющих системе тождеств Капелли некоторого порядка.

Развитию структурной теории алгебр произвольной конечной сигнатуры над полем произвольной характеристики или нётеровым коммутативно-ассоциативньм кольцом, удовлетворяющих системе тождеств Капелли некоторого порядка, посвящены работы [3]-[5]. В этих работах показано, что структурная теория таких алгебр является содержательной, и многие результаты о структуре ассоциативных PI-алгебр и конечномерных алгебр могут быть перенесены на случай алгебр произвольной конечной сигнатуры, удовлетворяющих тождествам Капелли некоторого порядка.

Работа выполнена при финансовой поддержке Российского фонда фундаментальных исследований (грант № 96-01-01088). 
В [1], [6], [7] было доказано, что радикал Джекобсона конечно порожденной PI-алгебры над произвольным нётеровым кольцом является нильпотентным идеалом. Поэтому в конечно порожденной ассоциативной РІ-алгебре над произвольньп нётеровым кольцом наибольший нильпотентный идеал существует и совпадает с радикалом Джекобсона.

Всякая конечномерная алгебра обладает наибольшим нильпотентньм идеалом. Основным результатом данной статьи является

ТЕОРемА 1. Пусть $L$ - конечно порожденная алгебра конечной сигнатурь $\Omega$ над полем произвольной характеристики или произвольным нётеровым коммутативно-ассочиативным кольцом $C$, и $C$-алгебра $L$ удовлетворяет тождествам Капелли некоторого порядка. Тогда в алгебре L существует наибольиий нильпотентный идеал $N(L)$.

При доказательстве этой теоремы сушественно используются комбинаторные методы, развитые в [2]-[5].

Теорема 1 допускает обобшение на пары (представления) сигнатуры конечного типа, удовлетворяющие слабым тождествам Капелли. Класс представлений конечного типа достаточно широк и включает в себя представления алгебр Ли, (специальных) йордановых алгебр, лиевых и суперлиевых тройных систем в ассоциативных алгебрах [3].

ТЕОРема 2. Пусть $(A, L)$ - конечно порожсденная пара $C$-алгебр сигнатуры конечного типа $\left(\Omega, \Omega^{\prime}\right)$, удовлетворяющая слабым тожсдествам Капелли некоторого порядка, и $C$ - произвольное поле или нётерово коммутативно-ассоциативное кольцо. Тогда в алгебре А существует наибольший нильпотентный идеал $N(A)$.

Обозначения в статье согласованы с обозначениями в [2]-[5].

\section{§1. Предварительные сведения и определения}

В этом параграфе будут сформулированы основные результаты из [3]-[5], касающиеся комбинаторных свойств тождеств Капелли и структурных свойств алгебр, удовлетворяющих тождествам Капелли.

Пусть $C$ - коммутативное и ассоциативное кольцо с единицей. Будем понимать под $C$-алгеброй сигнатурь $\Omega C$-модуль $A$, на котором для каждого $\omega \in \Omega$ определена $C$-полилинейная операция с арностью $n=n(\omega)$

$$
\omega: \underbrace{A \otimes_{C} A \otimes_{C} \cdots \otimes_{C} A}_{n \text { раз }} \rightarrow A .
$$

Для всякой $C$-алгебры $A$ сигнатуры $\Omega$ обозначим через $D(A)$ ассоциативную $C$-алгебру, которая порождена в $\operatorname{End}_{C} A$ единичным оператором и всеми элементами вида $\left.f\left(\widehat{x}_{0}, x_{1}, \ldots, x_{q}\right)\right|_{x_{1}=v_{1}, \ldots, x_{q}=v_{q}}$, где $f\left(x_{0}, \ldots, x_{q}\right)$ - полином из абсолютно свободной $C$-алгебры сигнатуры $\Omega$, который линеен по $x_{0}$, и по определению $f\left(\widehat{x}_{0}, v_{1}, \ldots, v_{q}\right) \times v=f\left(v, v_{1}, \ldots, v_{q}\right)$ для всех $v \in A$. Если $A$ - бинарная алгебра, то алгебра $D(A)$ называется алгеброй левых и правых умножений, ассочиированной с алгеброй $A$. 
Алгебра $A$ обладает канонической структурой левого $D(A)$-модуля. Эта структура задается естественным вложением алгебры $D(A)$ в алгебру $\operatorname{End}_{C} A$. Идеалами алгебры $A$ называют подмодули $D(A)$-модуля $A$.

Дополнительно будем предполагать, что среди сигнатурных операций алгебры $A$ имеется операция, арность которой $\geqslant 2$.

Будем говорить, что идеал $I$ алгебры $A$ сигнатуры $\Omega$ является произведением идеалов $I_{1}, \ldots, I_{q}$ алгебры $A$, если идеал $I$ порожден элементами вида

$$
\left.g\right|_{x_{1}=v_{1}, \ldots, x_{q}=v_{q}, \ldots, x_{r}=v_{r}},
$$

где $g\left(x_{1}, \ldots, x_{q}, \ldots, x_{r}\right)$ - полином из абсолютно свободной $C$-алгебры сигнатуры $\Omega$, полилинейный по $x_{1}, \ldots, x_{q}$, зависящий, быть может, от других переменных $x_{i}$, $q<i$. Кроме того, $v_{i} \in I_{i}, 1 \leqslant i \leqslant q$, и $v_{i} \in A$, если $i>q$. Будем писать в этом случае, что $I=\left(I_{1} \cdot \cdots . I_{q}\right)$. Определенное таким образом произведение идеалов является коммутативньм.

ОПредЕлЕНИЕ 1 . Идеал $I$ алгебры $A$ сигнатуры $\Omega$ называется нильпотентныцм класса п, если

$$
\underbrace{(I . \cdots . I)}_{n \text { раз }} \neq(0), \quad \underbrace{(I . \cdots . I)}_{n+1 \text { раз }}=(0) .
$$

Отметим, что сумма двух или любого конечного числа нильпотентных идеалов является нильпотентным идеалом, поскольку идеал $\sum_{i=1}^{m} I_{i}$, где $I_{i}, 1 \leqslant i \leqslant m,-$ нильпотентный идеал класса $n_{i}$ алгебры $A$ сигнатуры $\Omega$, нильпотентен в алгебре $A$ класса не вьше $\sum_{i=1}^{m} n_{i}$.

Здесь мы напомним некоторые определения и результаты из работ [2]-[5].

ЛЕмма 1. Для всякой алгебры $L$ сигнатуры $\Omega$ имножества переменных $x_{1}, x_{2}, \ldots$ существует алгебра $\bar{L}$ той же сигнатурь $\Omega$ такая, что

а) алгебра $L$ является подалгеброй алгебры $\bar{L}$, и алгебра $\bar{L}$ порождена алгеброй $L$ и әлементами $x_{1}, x_{2}, \ldots$;

б) любое отображение множества $\left\{x_{i} \mid i \in \mathbb{N}\right\}$ в алгебру $\bar{L}$ однозначно продолжается до гомоморфизма алгебры $\bar{L}$ в себя, тождественного на L

в) әлемент $f\left(x_{1}, \ldots, x_{q}\right) \in \bar{L}$, который является полиномом сигнатуры $\Omega$ от $x_{1}, \ldots, x_{q}$ u, возможно, от әлементов алгебры $L$, равен нулю тогда и только тогда, когда $f\left(v_{1}, \ldots, v_{q}\right)=0$ в L для любих $v_{1}, \ldots, v_{q} \in L$.

Алгебра $\bar{L}$ определена однозначно и является факторалгеброй свободного произведения свободной алгебры сигнатуры $\Omega$ со свободными образующими $x_{1}, x_{2}, \ldots$ и алгебры $L$ по идеалу обобшенных тождеств алгебры $L$.

Очевидно, что множества тождеств алгебры $L$ и $\bar{L}$ совпадают, т.е. $\operatorname{var} L=\operatorname{var} \bar{L}$.

Элементы алгебры $\bar{L}$ можно рассматривать как полиномы сигнатуры $\Omega$ от переменных типа $x\left(x_{i}, i \in \mathbb{N}\right)$ с коэффициентами из алгебры $L$.

Пусть в алгебре $L$ выполняются тождества Капелли порядка $n+1$.

Следуюшая лемма, доказанная в [4], представляет обобшение тождества Гамильтона-Кэли. 
ЛЕмма 2 (о тождестве Гамильтона-Кэли). Пусть $f\left(x_{1}, \ldots, x_{n}, x_{n+1}\right)-n o л u$ ном из $\bar{L}$, полилинейный по $x_{1}, \ldots, x_{n}, x_{n+1}$, кососимметричный по $x_{1}, \ldots, x_{n}$ и зависящий, быть мохсет, от других переменных типа $x, a$ - произвольныи әлемент из $D(\bar{L})$. Тогда в $\bar{L}$

$$
\left.\sum_{q=0}^{n}(-1)^{q} \sum_{1 \leqslant j_{1}<\cdots<j_{q} \leqslant n} f\right|_{x_{j_{1}}=a \times x_{j_{1}}, \ldots, x_{j_{q}}=a \times x_{j_{q}}, x_{n+1}=a^{n-q} \times x_{n+1}}=0
$$

В лемме 3 из [4] доказано тождество, показывающее, что можно переставлять два набора из $n$ "кососимметричных" переменных.

Лемма 3. Пусть $f\left(x_{1}, \ldots, x_{2 n}\right)$ - полином сигнатуры $\Omega$, который полилинеен по переменным $x_{1}, \ldots, x_{2 n}$, кососимметричен по переменным $x_{1}, \ldots, x_{n}$ и по переменным $x_{n+1}, \ldots, x_{2 n}$, зависит, быть может, от других переменных типа $x$. Тогда в алгебре $\bar{L}$ выполняется равенство

$$
f\left(x_{1}, \ldots, x_{n}, x_{n+1}, \ldots, x_{2 n}\right)-f\left(x_{n+1}, x_{n+2}, \ldots, x_{2 n}, x_{1}, x_{2}, \ldots, x_{n}\right)=0
$$

В [5] доказана

ТЕОРема 3. Пусть конечно порожденная $C$-алгебра L конечной сигнатуры удовлетворяет системе тожсдеств Капелли порядка $n+1$. Тогда существуют такие коммутативная и ассоциативная $C$-алгебра $\Phi$ с единицей и идеал $J$ $\Phi$-алгебрь $\Phi \otimes_{C} L$, что идеал $J \cap L$ алгебры $L$ нильпотентен класса не выше $n$, и в $C$-алгебре $\Phi$ существует такая конечно порожденная $C$-подалгебра $\Phi^{\prime}$, что $\Phi^{\prime}$-модуль $\Phi^{\prime} \cdot(L / L \cap J)$ конечно порохден.

Там же показано, что в качестве $\Phi$ можно взять кольцо полиномов $C\left[\sigma_{q}(a)\right.$ $a \in D(L), 1 \leqslant q \leqslant n]$, где $\sigma_{q}(a), a \in D(L), 1 \leqslant q \leqslant n$, являются свободными коммутирующими переменньми. Будем считать, что $\sigma_{0}(a)=1_{C}$ для всех $a \in D(L)$. В качестве $J$ можно взять идеал $\Phi$-алгебры $\Phi \otimes_{C} L$, порожденный элементами вида

$$
\sum_{q=0}^{n}(-1)^{q} \sigma_{q}(a) \otimes a^{n-q} \times v
$$

где $v \in L, a \in D(L)$.

Пусть $B_{n}$ - идеал алгебры $\bar{L}$, порожденный элементами вида

$$
\left.f\right|_{x_{1}=w_{1}, \ldots, x_{n}=w_{n}, x_{n+1}=w_{n+1}, \ldots}
$$

где $f$ - произвольньй элемент алгебры $\bar{L}$, который полилинеен и кососимметричен по переменным $x_{1}, \ldots, x_{n}$, возможно, зависит от других переменных типа $x$, а $w_{1}, \ldots, w_{n}, w_{n+1}, \ldots$ - произвольные элементы из алгебры $\bar{L}$. Из определения идеала $B_{n}$ следует, что алгебра $\bar{L} / B_{n}$ удовлетворяет системе тождеств Капелли порядка $n$. 
Пусть идеал $W_{n}$ алгебры $\bar{L}$ - это наименшший идеал алгебры $\bar{L}$ среди тех идеалов, которые

1) содержат все элементы вида $d_{n}\left(x_{1}, \ldots, x_{n}\right)$, где $d_{n}\left(x_{1}, \ldots, x_{n}\right)$ - элемент алгебры $\bar{L}$, полилинейный и кососимметричный по переменным $x_{1}, \ldots, x_{n}$, зависящий, быть может, от других переменных типа $x$;

2) замкнуты относительно всех гомоморфизмов алгебры $\bar{L}$ в себя, которые являются тождественным отображением на $L$ и переводят переменные типа $x$ в переменные типа $x$.

Отметим, что идеал $W_{n}$ содержит все элементы алгебры $\bar{L}$, полилинейные и кососимметричные по $n$ переменным типа $x$, и $W_{n} \subset B_{n}$.

Обозначим буквой $F$ кольцо полиномов $C\left[\sigma_{q}(a) \mid a \in D(\bar{L}), 1 \leqslant q \leqslant n\right]$, где $\sigma_{q}(a), a \in D(\bar{L}), 1 \leqslant q \leqslant n$, являются свободными коммутирующими переменными. Будем считать, что $\sigma_{0}(a)=1_{C}$ для всех $a \in D(\bar{L})$.

Пусть $\bar{J}$ - идеал $F$-алгебры $F \otimes_{C} \bar{L}$, порожденный элементами вида

$$
\sum_{q=0}^{n}(-1)^{q} \sigma_{q}(a) \otimes a^{n-q} \times v,
$$

где $v \in \bar{L}, a \in D(\bar{L})$.

Тогда $\Phi \hookrightarrow F, J \hookrightarrow \bar{J}$.

Лемма 4 из [5], хотя и техническая по своему характеру, представляет самостоятельный интерес. Здесь показано, как для каждого $b \in D(\bar{L})$ построить отображение $\varphi(b) \in \operatorname{End}_{D(\bar{L})} F \otimes_{C} W_{n}$, задаюшее структуру $C\left[\sigma_{p}(b) \mid 1 \leqslant p \leqslant n\right]$-модуля на $W_{n}$.

Лемма 4. Для всякого әлемента $b$ алгебры $D(\bar{L})$ существует такой әлемент $\varphi(b)$ из $\operatorname{End}_{D(\bar{L})} F \otimes_{C} W_{n}$ - алгебры всех эндоморфизмов $D(\bar{L})$-модуля $F \otimes_{C} W_{n}$, чmo

1) $\left.\varphi(b)\right|_{F(b) \otimes_{C} W_{n}}=\mathrm{id}$, əде $F(b)=C\left[\sigma_{p}(a) \mid a \in D(\bar{L}), a \neq b, 0 \leqslant p \leqslant n\right]$, т.е. $F(b)$ - это $C$-подалгебра алгебры $F$, которая состоит из әлементов, не зависящих от $\sigma_{p}(b), 1 \leqslant p \leqslant n$;

2) отображение $\varphi(b)$ задает на $W_{n}$ структуру модуля над $C\left[\sigma_{p}(b) \mid\right.$ $0 \leqslant p \leqslant n]$ следующим образом: $f \cdot d=\varphi(b)(f \otimes d)$, где $f \in C\left[\sigma_{p}(b) \mid\right.$ $0 \leqslant p \leqslant n], d \in W_{n}$ (здесь предполагается, что $W_{n} \hookrightarrow F \otimes_{C} W_{n}$ посредством $d \rightarrow 1 \otimes d)$;

3) если $f_{1} \in F(b), f_{2} \in C\left[\sigma_{p}(b) \mid 0 \leqslant p \leqslant n\right], \operatorname{mo} \varphi(b)\left(f_{1} f_{2} \otimes d\right)=f_{1} \otimes f_{2} \cdot d$, əде $d \in W_{n}$

4) если полином $g\left(x_{0}, x_{1}, \ldots, x_{r}\right)$ сигнатуры $\Omega$ полилинеен по переменныцм $x_{0}, x_{1}, \ldots, x_{n}$, кососимметричен по переменным $x_{1}, \ldots, x_{n}$ и зависит, бъть мохет, от других переменных типа $x$, то

$$
\varphi(b)\left(\sum_{j=0}^{n}(-1)^{j} \sigma_{j}(b) \otimes g\left(b^{n-j} \times v, x_{1}, \ldots, x_{r}\right)\right)=0
$$

в идеале $W_{n}$, где $v \in \bar{L}$. 
Из условий 1)-3) получаем, что для задания эндоморфизма $\varphi(b)$ достаточно определить на $W_{n}$ структуру модуля над $C\left[\sigma_{p}(b) \mid 0 \leqslant p \leqslant n\right]$, причем действие алгебры $C\left[\sigma_{p}(b) \mid 0 \leqslant p \leqslant n\right]$ на $W_{n}$ должно коммутировать с действием алгебры $D(\bar{L})$.

В [5] показано, что такое действие элементов $\sigma_{p}(b), 0 \leqslant p \leqslant n$, на элементы $d_{n}\left(x_{1}, \ldots, x_{n}\right)$ идеала $W_{n}$, которые полилинейны и кососимметричны по переменным $x_{1}, \ldots, x_{n}$, можно задать следуюшим образом:

$$
\sigma_{p}(b) \cdot d_{n}\left(x_{1}, \ldots, x_{n}\right)=\left.\sum_{1 \leqslant j_{1}<\cdots<j_{p} \leqslant n} d_{n}\right|_{x_{j_{1}}=b \times x_{j_{1}}, \ldots, x_{j_{p}}=b \times x_{j_{p}}}
$$

где $1 \leqslant p \leqslant n,\left.\sigma_{0}(b)\right|_{W_{n}}=\mathrm{id}$.

На остальные элементы идеала $W_{n}$ действие элементов $\sigma_{p}(b), 0 \leqslant p \leqslant n$, продолжается посредством специализации переменных типа $x$ в переменные типа $x$. Корректность определения действия следует из леммы 3 , выполнение условия 4) из леммы 2.

\section{§2. Доказательство основной теоремы 1}

В этом параграфе будет доказана лемма 5 и основная теорема 1.

ЛЕмма 5. Пусть в алгебре $L$ конечной сигнатуры $\Omega$ над нётеровым коммутативно-ассоциативным кольцом $C$ выполняются тождества Капелли порядка $n+1$. Тогда $\left(W_{n} . \bar{J}\right) \cap \bar{L}=(0)$.

ДоказАтельство. Пусть $d \in\left(W_{n} \cdot \bar{J}\right) \cap \bar{L}$. Тогда для любого $b \in D(\bar{L})$ получаем, что $\varphi(b)(d)=d$.

Для всякого $b \in D(\bar{L})$ определим $\bar{J}(b)$ - идеал $F$-алгебры $F \otimes_{C} \bar{L}$, порожденньй элементами вида

$$
\sum_{q=0}^{n}(-1)^{q} \sigma_{q}(b) \otimes b^{n-q} \times v
$$

где $v \in \bar{L}$, а $b$ - фиксированный элемент из $D(\bar{L})$. Тогда

$$
d \in \sum_{i=1}^{r}\left(W_{n} \cdot \bar{J}\left(b_{i}\right)\right)
$$

для некоторых $b_{1}, \ldots, b_{r} \in D(\bar{L})$. Из условия 4) леммы 4 получаем, что для $\psi=$ $\varphi\left(b_{1}\right) \cdots \varphi\left(b_{r}\right)$ верно

$$
\psi(d)=0
$$

Таким образом, $d=\psi(d)=0$. Поэтому $d=0$. Лемма доказана.

Доказательство теоремы 1 будет проведено индукцией по порядку тождеств Капелли.

База индукции. Утверждение теоремы 1 верно для алгебр, удовлетворяющих тождествам Капелли порядка 2. 
Доказательство базы индукции. Пусть в конечно порожденной алгебре $L$ конечной сигнатуры $\Omega$ над произвольным полем или нётеровым коммутативно-ассоциативным кольцом $C$ вьполняются тождества Капелли порядка 2. Тогда $B_{1}=\bar{L}$, $x_{1} \in W_{1}$.

Пусть $N_{0} \subset N_{1} \subset \cdots \subset N_{t} \subset \cdots$ - бесконечная строго возрастаюшая цепочка нильпотентных идеалов алгебры $L$. Докажем, что идеал $\widetilde{N}=\bigcup_{t=0}^{\infty} N_{t}-$ нильпотентньй идеал алгебры $L$.

Из теоремы 3 следует, что для некоторого $m$

$$
\Phi^{\prime} \otimes_{C} \widetilde{N} \subseteq \Phi^{\prime} \otimes_{C} N_{m}+J
$$

(из нётеровости $\Phi^{\prime}$-модуля $\left.\Phi^{\prime} \cdot(L / L \cap J)\right)$. Обозначим через $\bar{N}_{m}$ идеал в $\bar{L}$, порожденный $N_{m}$. Пусть $q$-класс нильпотентности идеала $N_{m}$. Тогда $q$ также и класс нильпотентности идеала $\bar{N}_{m}$.

Пусть $f\left(x_{1}, \ldots, x_{q+2}, \ldots\right)$ - моном из $\bar{L}$, полилинейный по $x_{1}, \ldots, x_{q+2}$, зависящий, быть может, от других переменных типа $x$.

Пусть $v_{1}, \ldots, v_{q+2} \in \widetilde{N}$. Тогда $v_{i}=v_{i}^{\prime}+h_{i}$, где $v_{i}^{\prime} \in \Phi^{\prime} \otimes_{C} N_{m}, h_{i} \in J$, $1 \leqslant i \leqslant q+2$. Тогда

$$
\left.f\right|_{x_{2}=v_{2}, \ldots, x_{q+2}=v_{q+2}} \in(\Phi^{\prime} \otimes_{C} \underbrace{\left(\bar{N}_{m} \cdot \cdots \cdot \bar{N}_{m}\right)}_{q+1 \text { раз }}+\left(W_{1} . J\right)) \cap \bar{L} .
$$

Так как идеал $\bar{N}_{m}$ нильпотентен класса $q$, получаем

$$
\underbrace{\left(\bar{N}_{m} \cdot \cdots \cdot \bar{N}_{m}\right)}_{q+1 \text { раз }}=(0) \text {. }
$$

Из леммы 5 получаем, что $\left(W_{1} . J\right) \cap \bar{L}=(0)$. Поэтому

$$
\left.f\right|_{x_{2}=v_{2}, \ldots, x_{q+2}=v_{q+2}}=0
$$

в $\bar{L}$, а значит, и

$$
\left.f\right|_{x_{1}=v_{1}, x_{2}=v_{2}, \ldots, x_{q+2}=v_{q+2}}=0
$$

в $\bar{L}$.

Это означает, что идеал $\widetilde{N}$ алгебры $L$ нильпотентен класса не выше $q+1$. По лемме Цорна среди нильпотентных идеалов алгебры $L$ сушествует наибольший. Основание индукции доказано.

Шаг индукции. Пусть утверждение теоремы 1 верно для всех алгебр, удовлетворяющих тождествам Капелли порядка $n$, и в конечно порожденной алгебpe $L$ конечной сигнатуры $\Omega$ над произвольным полем или нётеровым коммутативно-ассоциативньм кольцом $C$ выполняются тождества Капелли порядка $n+1$.

По предположению индукции в алгебре $L / L \cap B_{n}$ сушествует наибольший нильпотентньй идеал $N\left(L / L \cap B_{n}\right)$. Пусть $r$ класс нильпотентности этого идеала. 
Пусть $N_{0} \subset N_{1} \subset \cdots \subset N_{t} \subset \cdots$ - бесконечная строго возрастающая цепочка нильпотентных идеалов алгебры $L$. Докажем, что идеал $\widetilde{N}=\bigcup_{t=0}^{\infty} N_{t}-$ нильпотентньй идеал алгебры $L$.

Из теоремы 3 следует, что для некоторого $m$

$$
\Phi^{\prime} \otimes_{C} \tilde{N} \subseteq \Phi^{\prime} \otimes_{C} N_{m}+J
$$

(из нётеровости $\Phi^{\prime}$-модуля $\left.\Phi^{\prime} \cdot(L / L \cap J)\right)$. Обозначим через $\bar{N}_{m}$ идеал в $\bar{L}$, порожденный $N_{m}$. Пусть $q$ - класс нильпотентности идеала $N_{m}$. Тогда $q$ также и класс нильпотентности идеала $\bar{N}_{m}$.

Из индуктивного предположения получаем, что

$$
\underbrace{(\tilde{N} . \cdots . \tilde{N})}_{r+1 \text { раз }} \subseteq L \cap B_{n} .
$$

Пусть $f\left(x_{1}, \ldots, x_{l}, \ldots\right)$ - моном из $\bar{L}$, полилинейный по всем своим переменным типа $x$, среди которых имеются $x_{1}, \ldots, x_{l}$, зависящий, быть может, от других переменных типа $x$. Докажем, что сушествует достаточно большое $l$, что для всякого такого монома

$$
\left.f\left(x_{1}, \ldots, x_{l}, \ldots\right)\right|_{x_{1}=v_{1}, \ldots, x_{l}=v_{l}}=0
$$

в $\bar{L}$, где $v_{1}, \ldots, v_{l} \in \widetilde{N}$.

Сушествует достаточно большое $l$, что всякий такой моном $f$ после перенумерации переменных $x_{1}, \ldots, x_{l}$ имеет вид

$$
f_{1}\left(f_{2}\left(x_{1}, \ldots, x_{s}, \ldots\right), x_{s+1}, \ldots, x_{l}, \ldots\right)
$$

где $r+1 \leqslant s \leqslant l, l-s \geqslant q+1$, и $f_{2}\left(x_{1}, \ldots, x_{s}, \ldots\right)$ - моном из $\bar{L}$, полилинейный по $x_{1}, \ldots, x_{s}$, зависяший, быть может, от других переменных типа $x$, $f_{1}\left(t, x_{s+1}, \ldots, x_{l}, \ldots\right)$ - моном из $\bar{L}$, полилинейный по $t, x_{s+1}, \ldots, x_{l}$, зависящий, быть может, от других переменных типа $x$, а $t$ - некоторая переменная типа $x$.

В качестве $l$ можно взять $k \cdot r+q+1$, где $k$-максимальная “арность" операции из $\Omega$. Отметим, что $l$ зависит только от $r, q$ и максимальной “арности” операции из $\Omega$, но не зависит от числа порождающих алгебры $L$, мощности сигнатуры $\Omega$, порядка тождеств Капелли $n+1$.

Пусть $v_{1}, \ldots, v_{l} \in \widetilde{N}$, а $v_{l+1}, \cdots \in L$. Тогда $v_{i}=v_{i}^{\prime}+h_{i}$, где $v_{i}^{\prime} \in \Phi^{\prime} \otimes_{C} N_{m}$, $h_{i} \in J, s+1 \leqslant i \leqslant l$. Тогда

$$
\bar{f}=\left.f_{2}\right|_{x_{1}=v_{1}, \ldots, x_{s}=v_{s}, x_{l+1}=v_{l+1}, \ldots} \in B_{n} \cap L
$$

Тогда элемент $\bar{f}$ из алгебры $L$ может быть представлен в виде $C$-линейной комбинации элементов вида $\left.g\left(t_{1}, \ldots, t_{n}\right)\right|_{t_{1}=w_{1}, \ldots, t_{n}=w_{n}}$, где полином $g\left(t_{1}, \ldots, t_{n}\right)$ из алгебры $\bar{L}$ полилинеен и кососимметричен по переменным $t_{1}, \ldots, t_{n}$, переменные $t_{1}, \ldots, t_{n}$ являются переменными типа $x$, т.е. $g\left(t_{1}, \ldots, t_{n}\right) \in W_{n}$, элементы $w_{1}, \ldots, w_{n}$ лежат в алгебре $L$. 
Будем считать, что среди переменных $t_{1}, \ldots, t_{n}$ нет переменных, от которых зависит моном $f$.

Поэтому

$$
\left.f\right|_{x_{1}=v_{1}, \ldots, x_{s}=v_{s}, x_{l+1}=v_{l+1}, \ldots}=\left.\left(\left.f_{1}\right|_{t=\bar{f}}\right)\right|_{x_{l+1}=v_{l+1}, \ldots}
$$

может быть представлен в виде $C$-линейной комбинации элементов вида

$$
\left.\left(\left.\left(\left.f_{1}\right|_{t=g}\right)\right|_{x_{l+1}=v_{l+1}, \ldots}\right)\right|_{t_{1}=w_{1}, \ldots, t_{n}=w_{n}}
$$

где полином $g\left(t_{1}, \ldots, t_{n}\right)$ из алгебры $\bar{L}$ полилинеен и кососимметричен по переменным $t_{1}, \ldots, t_{n}$, переменные $t_{1}, \ldots, t_{n}$ являются переменными типа $x$, т.е. $g\left(t_{1}, \ldots, t_{n}\right) \in W_{n}$, элементы $w_{1}, \ldots, w_{n}$ лежат в алгебре $L$.

Подставим в полином $\left.f_{1}\right|_{t=g}$ вместо переменных $x_{s+1}, \ldots, x_{l}$ элементы $v_{s+1}, \ldots, v_{l}$ из $\widetilde{N}$. Поскольку $v_{i}=v_{i}^{\prime}+h_{i}$, где $v_{i}^{\prime} \in \Phi^{\prime} \otimes_{C} N_{m}, h_{i} \in J$, $s+1 \leqslant i \leqslant l$, получим, что

$$
\left.f_{1}\right|_{t=g, x_{s+1}=v_{s+1}, \ldots, x_{l}=v_{l}} \in(\Phi^{\prime} \otimes_{C} \underbrace{\left(\bar{N}_{m} . \cdots \cdot \bar{N}_{m}\right)}_{q+1 \text { раз }}+\left(W_{n} . J\right)) \cap \bar{L} .
$$

Так как идеал $\bar{N}_{m}$ нильпотентен класса $q$, получим

$$
\underbrace{\left(\bar{N}_{m} \cdot \cdots \cdot \bar{N}_{m}\right)}_{q+1 \text { раз }}=(0) .
$$

Из леммы $5\left(W_{n} . J\right) \cap \bar{L}=(0)$. Поэтому

$$
\begin{gathered}
\left.f_{1}\right|_{t=g, x_{s+1}=v_{s+1}, \ldots, x_{l}=v_{l}}=0 \\
\left.\left(\left.f_{1}\right|_{t=g, x_{s+1}=v_{s+1}, \ldots, x_{l}=v_{l}, \ldots}\right)\right|_{t_{1}=w_{1}, \ldots, t_{n}=w_{n}}=0 \\
\left.f_{1}\right|_{t=\bar{f}, x_{s+1}=v_{s+1}, \ldots, x_{l}=v_{l}}=0 \\
\left.f\right|_{x_{1}=v_{1}, \ldots, x_{l}=v_{l}}=0
\end{gathered}
$$

Таким образом, доказано, что идеал $\widetilde{N}$ нильпотентен класса меньше $l$. По лемме Цорна среди нильпотентных идеалов алгебры $L$ сушествует наибольший.

Теорема 1 доказана. 


\section{Список литературы}

1. Размыслов Ю. П. О радикале Джекобсона в РІ-алгебрах // Алгебра и логика. 1974. T. 13. №3. C. 337-360.

2. Размыслов Ю. П. Алгебры, удовлетворяющие тождественњым соотношениям типа Капелли // Изв. АН СССР. Сер. матем. 1981. Т. 45. № 1. С. 143-166.

3. Razmyslov Yu.P., Zubrilin K. A. Capelli identities and representations of finite type // Comm. Algebra. 1994. V. 22 (14). P. 5733-5744.

4. Зубрилин К. А. Алгебры, удовлетворяющие тождествам Капелли // Матем. сб. 1995. T. 186. №3. C. 53-64.

5. Зубрилин $K . A$. О классе нильпотентности препятствия для представимости алгебр, удовлетворяющих тождествам Капелли // Фундамент. и прикл. матем. 1995. Т. 1. № 2 . C. 409-430.

6. Кемер А.Р. Тождества Капелли и нильпотентность радикала Джекобсона конечно порожденной PI-алгебры // Докл. АН СССР. 1980. Т. 245. № 4. С. 793-799.

7. Braun A. The Jacobson radical in a finitely generated P.I. algebra // Bull. Amer. Math. Soc. 1982. V. 7. № 2. P. 385-386.

Московский государственньй

Поступила в редакцию университет им. М.В. Ломоносова

08.10 .1996 